Commun. Korean Math. Soc. 25 (2010), No. 1, pp. 51-57

DOI 10.4134/CKMS.2010.25.1.051

\title{
THE LACUNARY STRONG ZWEIER CONVERGENT SEQUENCE SPACES
}

\author{
MEHMET ŞENGÖNÜL
}

\begin{abstract}
In this paper we introduce and study the lacunary strong Zweier sequence spaces $N_{\theta}^{0}[\mathcal{Z}], N_{\theta}[\mathcal{Z}]$ consisting of all sequences $x=\left(x_{k}\right)$ such that $(Z x)$ in the space $N_{\theta}$ and $N_{\theta}^{0}$ respectively, which is normed. Also, prove that $N_{\theta}^{0}[\mathcal{Z}], N_{\theta}[\mathcal{Z}]$ are linearly isomorphic to the space $N_{\theta}^{0}$ and $N_{\theta}$, respectively. And we study some connections between lacunary strong Zweier sequence and lacunary statistical Zweier convergence sequence.
\end{abstract}

\section{Introduction}

A sequence space $\lambda$ with linear topology is called a $K$-space provided each of maps $p_{i} \rightarrow \mathbb{C}$ defined by $p_{i}(x)=x_{i}$ is continuous for all $i \in \mathbb{N}$; where $\mathbb{C}$ denotes the complex field and $\mathbb{N}=\{0,1,2, \ldots\}$. A $K$-space $\lambda$ is called an $F K$-space provided $\lambda$ is a complete linear metric space. An $F K$-space whose topology is normable is called a $B K$-space [2].

For a sequence space $\lambda$, the matrix domain $\lambda_{A}$ of an infinite matrix $A$ is defined by

$$
\lambda_{A}=\left\{x=\left(x_{k}\right) \in w: A x \in \lambda\right\},
$$

where $A=\left(a_{n k}\right)(n, k=0,1,2, \ldots)$ is an infinite matrix of complex numbers and $A x=\left(A_{n}(x)\right)$ if $A_{n}(x)=\sum_{k} a_{n k} x_{k}$ converges for each $n$.

By a lacunary sequence we mean an increasing integer sequence $\theta=\left(k_{r}\right)$ such that $k_{0}=0$ and $h_{r}=k_{r}-k_{r-1} \rightarrow \infty$ as $r \rightarrow \infty$. In this paper the intervals determined by $\theta$ will be denoted by $I_{r}=\left(k_{r-1}, k_{r}\right]$ and also the ratio $\frac{k_{r}}{k_{r-1}}$ will be abridged by $q_{r}$. The space of lacunary convergence sequences $N_{\theta}$ was defined by Freedman et al. [5], as follows:

$$
N_{\theta}=\left\{x=\left(x_{i}\right) \in w: \lim _{r \rightarrow \infty} \frac{1}{h_{r}} \sum_{i \in I_{r}}\left|x_{i}-\ell\right|=0 \text { for some } \ell\right\} .
$$

Received September 12, 2008.

2000 Mathematics Subject Classification. Primary 40C05, 40J05, 46A45.

Key words and phrases. lacunary sequence, Zweier space, statisticial convergence, Banach space, isomorphism.

(C)2010 The Korean Mathematical Society 
The space $N_{\theta}$ is a BK-space with the norm

$$
\|x\|_{N_{\theta}}=\sup _{r} \frac{1}{h_{r}} \sum_{i \in I_{r}}\left|x_{i}\right| .
$$

$N_{\theta}^{0}$ denotes the subset of $N_{\theta}$ those sequences for which $\ell=0$ in the definition $N_{\theta}$. Also; $\left(N_{\theta}^{0},\|\cdot\|_{N_{\theta}}\right)$ is a $B K$-space.

Let $c$ and $c_{0}$ be the linear spaces of bounded, convergent and null sequences with complex terms, respectively.

In [9], Şengönül introduced $\mathcal{Z}$ and $\mathcal{Z}_{0}$ spaces as the set of all sequences such that $Z$-transforms of them are in the spaces $c$ and $c_{0}$, respectively, i.e.,

$$
\mathcal{Z}=\left\{x=\left(x_{k}\right) \in w: Z x \in c\right\}
$$

and

$$
\mathcal{Z}_{0}=\left\{x=\left(x_{k}\right) \in w: Z x \in c_{0}\right\},
$$

where $Z=\left(z_{n k}\right)(n, k=0,1,2, \ldots)$ denotes by the matrix

$$
z_{n k}=\left\{\begin{array}{cc}
\frac{1}{2}, & k \leq n \leq k+1 \\
0, & \text { otherwise }
\end{array} \quad(n, k \in \mathbb{N}) .\right.
$$

This matrix is called Zweier matrix.

The purpose of this paper is to introduce and study the concept of lacunary strong Zweier convergence in the same way obtaining the sequence space $\mathcal{Z}$ from the sequence space $c$.

\section{Lacunary strong Zweier convergence}

We introduce the sequence spaces $N_{\theta}^{0}[\mathcal{Z}], N_{\theta}[\mathcal{Z}]$ and $\ell_{\infty}[\mathcal{Z}]$ as the set of all sequences such that $Z$-transforms of them are in the $N_{\theta}^{0}, N_{\theta}$ and $N_{\theta}^{\infty}$, respectively, that is

$$
N_{\theta}^{0}[\mathcal{Z}]=\left\{x=\left(x_{i}\right) \in w: \lim _{r} \frac{1}{h_{r}} \sum_{i \in I_{r}}\left|\frac{1}{2}\left(x_{i}+x_{i-1}\right)\right|=0\right\},
$$

$$
N_{\theta}[\mathcal{Z}]=\left\{x=\left(x_{i}\right) \in w: \lim _{r} \frac{1}{h_{r}} \sum_{i \in I_{r}}\left|\frac{1}{2}\left(x_{i}+x_{i-1}\right)-\ell\right|=0\right\},
$$

and

$$
N_{\theta}^{\infty}[\mathcal{Z}]=\left\{x=\left(x_{i}\right) \in w: \sup _{r} \frac{1}{h_{r}} \sum_{i \in I_{r}}\left|\frac{1}{2}\left(x_{i}+x_{i-1}\right)\right|<\infty\right\} .
$$

The $Z=\left(z_{n k}\right)_{n, k>0}$ matrix is well-known as a regular matrix, [1]. With the notation of (1), we may redefine the spaces $N_{\theta}^{0}[\mathcal{Z}], N_{\theta}^{0}[\mathcal{Z}]$ and $\ell_{\infty}[\mathcal{Z}]$ as follows:

$$
N_{\theta}^{0}[\mathcal{Z}]=\left(N_{\theta}^{0}\right)_{Z}, N_{\theta}^{0}[\mathcal{Z}]=\left(N_{\theta}\right)_{Z} \text { and } N_{\theta}^{\infty}[\mathcal{Z}]=\left(N_{\theta}^{\infty}\right)_{Z} .
$$


Zwei mean is two in German language. So, we called lacunary strong Zweier convergent sequence spaces(or lacunary statistical Zweier convergent sequence space) are the spaces $N_{\theta}^{0}[\mathcal{Z}], N_{\theta}[\mathcal{Z}], \ell_{\infty}[\mathcal{Z}]$ (or $S_{\theta}[\mathcal{Z}]$ ).

Define the sequence $y$ which will be frequently used, as $Z$-transform of a sequence $x$, i.e.,

$$
y_{i}=\frac{1}{2}\left(x_{i}+x_{i-1}\right) ;(i \in \mathbb{N}) .
$$

Theorem 2.1. The sets $N_{\theta}^{0}[\mathcal{Z}], N_{\theta}[\mathcal{Z}]$ and $N_{\theta}^{\infty}[\mathcal{Z}]$ are the linear spaces with the co-ordinatewise addition and scalar multiplication which are the BK-spaces with the norm $\|x\|_{N_{\theta}^{0}[\mathcal{Z}]}=\|x\|_{N_{\theta}[\mathcal{Z}]}=\|x\|_{N_{\theta}^{\infty}[\mathcal{Z}]}=\|Z x\|_{N_{\theta}}$.

Proof. The first part of the theorem is a routine verification and so we omit it. Furthermore, since (7) holds and $N_{\theta}^{0}, N_{\theta}$ are $B K$-spaces with respect to the norm defined by (3) [5] and the matrix $Z=\left(z_{n k}\right)$ is normal, i.e., $z_{n k} \neq 0$ for $0 \leq k \leq n$ and $z_{n k}=0$ for $k>n$ for all $n, k \in \mathbb{N}$ and also of Theorem 4.3.2 of Wilansky [10] gives the fact that the spaces $N_{\theta}^{0}[\mathcal{Z}], N_{\theta}^{0}[\mathcal{Z}]$ are the $B K$ spaces.

Theorem 2.2. The sequence spaces $N_{\theta}^{0}[\mathcal{Z}], N_{\theta}[\mathcal{Z}]$ and $N_{\infty}[\mathcal{Z}]$ are linearly isomorphic to the spaces $N_{\theta}^{0}, N_{\theta}$ and $N_{\infty}$ respectively, i.e., $N_{\theta}^{0}[\mathcal{Z}] \cong N_{\theta}^{0}$, $N_{\theta}[\mathcal{Z}] \cong N_{\theta}$ and $N_{\theta}^{\infty}[\mathcal{Z}] \cong N_{\theta}^{\infty}$.

Proof. We consider only $N_{\theta}^{0}[\mathcal{Z}]$. We should show the existence of a linear bijection between the spaces $N_{\theta}^{0}[\mathcal{Z}]$ and $N_{\theta}^{0}$. Consider the transformation $Z$ define, with the notation of $(8)$, from $N_{\theta}^{0}[\mathcal{Z}]$ to $N_{\theta}^{0}$ by

$$
\begin{aligned}
Z: N_{\theta}^{0}[\mathcal{Z}] & \longmapsto N_{\theta}^{0} \\
x & \longmapsto Z x=y, \quad y=\left(y_{i}\right), \quad y_{i}=\frac{1}{2}\left(x_{i}+x_{i-1}\right),(i \in \mathbb{N}) .
\end{aligned}
$$

The linearity of $Z$ is clear. Further, it is trivial that $x=0$ whenever $Z x=0$ and hence $Z$ is injective. Let $y \in N_{\theta}^{0}$ and define the sequence $x=\left(x_{k}\right)$ by

$$
x_{i}=2 \sum_{k=0}^{i}(-1)^{i-k} y_{k}(n \in \mathbb{N}) \text {. }
$$

Then

$$
\begin{aligned}
\|x\|_{N_{\theta}^{0}[\mathcal{Z}]} & =\lim _{r} \frac{1}{h_{r}} \sum_{i \in I_{r}}\left|\frac{1}{2}\left(x_{i}+x_{i-1}\right)\right| \\
& =\lim _{r} \frac{1}{h_{r}} \sum_{i \in I_{r}}\left|\frac{1}{2}\left(2 \sum_{k=0}^{i}(-1)^{i-k} y_{k}+2 \sum_{k=0}^{i-1}(-1)^{(i-1)-k} y_{k}\right)\right| \\
& =\sup _{r} \frac{1}{h_{r}} \sum_{i \in I_{r}}\left|y_{i}\right|
\end{aligned}
$$


which says us that $x \in N_{\theta}^{0}[\mathcal{Z}]$. Additionally, we observe that

$$
\begin{aligned}
\|x\|_{N_{\theta}^{0}[\mathcal{Z}]} & =\sup _{r} \frac{1}{h_{r}} \sum_{i \in I_{r}}\left|\frac{1}{2}\left(x_{i}+x_{i-1}\right)\right| \\
& =\sup _{r} \frac{1}{h_{r}} \sum_{i \in I_{r}}\left|\frac{1}{2}\left(2 \sum_{k=0}^{i}(-1)^{i-k} y_{k}+2 \sum_{k=0}^{i-1}(-1)^{(i-1)-k} y_{k}\right)\right| \\
& =\sup _{r} \frac{1}{h_{r}} \sum_{i \in I_{r}}\left|y_{i}\right|=\|y\|_{N_{\theta}^{0}} .
\end{aligned}
$$

Thus, we have that $x \in N_{\theta}^{0}[\mathcal{Z}]$ and consequently $Z$ is surjective. Hence, $Z$ is linear bijection which therefore says us that the spaces $N_{\theta}^{0}[\mathcal{Z}]$ and $N_{\theta}^{0}$ are linearly isomorphic, as was desired. It is clear here that if the spaces $N_{\theta}^{0}[\mathcal{Z}]$ and $N_{\theta}^{0}$ replaced by the spaces $N_{\theta}[\mathcal{Z}]$ and $N_{\theta}$ or $N_{\theta}^{\infty}[\mathcal{Z}]$ and $N_{\theta}^{\infty}$, respectively. Then we obtain the fact that $N_{\theta}[\mathcal{Z}] \cong N_{\theta}$ or $N_{\theta}^{\infty}[\mathcal{Z}] \cong N_{\theta}^{\infty}$. This completes proof.

There is a relation between $N_{\theta}$ and the space $\left|\sigma_{1}\right|$ of strong Cesaro summable sequences defined by

$$
\left|\sigma_{1}\right|=\left\{x=\left(x_{i}\right) \in w: \lim _{n} \frac{1}{n} \sum_{i=1}^{n}\left|x_{i}-\ell\right|=0 \text { for some } \ell\right\} .
$$

Clearly, in special case $\theta=\left(2^{r}\right)$, we have $N_{\theta}=\left|\sigma_{1}\right|[6]$.

Also we see that, there are strong connection between $N_{\theta}[\mathcal{Z}]$ and the sequence space $w[\mathcal{Z}]$, which is defined by

$$
w[\mathcal{Z}]=\left\{x=\left(x_{i}\right): \lim _{n \rightarrow \infty} \frac{1}{n} \sum_{i \in I_{r}}\left|\frac{1}{2}\left(x_{i}+x_{i-1}\right)-\ell\right|=0\right\} .
$$

Clearly, in special case $\theta=\left(2^{r}\right)$, we have $N_{\theta}[\mathcal{Z}]=w[\mathcal{Z}]$. Now we give some inclusion relations between these sequence spaces.

Hereafter, we write $y_{i}$ for $\frac{1}{2}\left(x_{i}+x_{i-1}\right)$.

Theorem 2.3. Let $\theta=\left(k_{r}\right),(r=1,2,3, \ldots)$ be a lacunary sequence. If $\liminf q_{r}>1$, then $w[\mathcal{Z}] \subseteq N_{\theta}[\mathcal{Z}]$.

Proof. Let $x \in w[\mathcal{Z}]$ and $\liminf q_{r}>1$. Then, there exits $\gamma>0$ such that $q_{r}=\frac{k_{r}}{k_{r-1}} \geq 1+\gamma$ for sufficiently large $r$. We can also choose a sufficiently large number $\mathrm{r}$, that

$$
\frac{h_{r}}{k_{r}} \geq \frac{\gamma}{\gamma+1} \text { and } \frac{k_{r}}{h_{r}} \geq \frac{\gamma+1}{\gamma} .
$$

Then,

$$
\frac{1}{k_{r}} \sum_{i=1}^{k_{r}}\left|y_{i}-\ell\right| \geq \frac{1}{k_{r}} \sum_{i \in I_{r}}\left|y_{i}-\ell\right|=\frac{h_{r}}{k_{r} h_{r}} \sum_{i \in I_{r}}\left|y_{i}-\ell\right| \geq \frac{\gamma}{(1+\gamma) h_{r}} \sum_{i \in I_{r}}\left|y_{i}-\ell\right|
$$


which yields that $x \in N_{\theta}[\mathcal{Z}]$.

Theorem 2.4. For $\lim \sup q_{r}<\infty$, we have $N_{\theta}[\mathcal{Z}] \subseteq w[\mathcal{Z}]$.

Proof. If $\lim \sup q_{r}<\infty$, then there exits $K>0$ such that $q_{r}<K$ for every $r$. Now suppose that $\epsilon>0$ and $x \in N_{\theta}[\mathcal{Z}]$. There exits $m_{0}$ such that for every $m \geq m_{0}$,

$$
H_{m}=\frac{1}{h_{m}} \sum_{i \in I_{r}}\left|y_{i}-\ell\right|<\epsilon .
$$

We can also find $T>0$ such that $H_{m} \leq T$ for all $m$. Let $n$ be any integer with $k_{r} \geq n>k_{r-1}$. Now write

$$
\begin{aligned}
\frac{1}{n} \sum_{i=1}^{n}\left|y_{i}-\ell\right| & \leq \frac{1}{k_{r}} \sum_{i=1}^{k_{r}}\left|y_{i}-\ell\right| \\
& =\frac{1}{k_{r-1}} \sum_{m=1}^{m_{0}} \sum_{i \in I_{m}}\left|y_{i}-\ell\right|+\frac{1}{k_{r-1}} \sum_{m=m_{0}+1}^{k_{r}} \sum_{i \in I_{m}}\left|y_{i}-\ell\right| \\
& \leq \frac{1}{k_{r-1}} \sum_{m=1}^{m_{0}} \sum_{i \in I_{m}}\left|y_{i}-\ell\right|+\frac{\epsilon\left(k_{r}-k_{m_{0}}\right)}{k_{r-1}} \\
& \leq \frac{1}{k_{r-1}} \sum_{m=1}^{m_{0}} h_{i} H_{i}+\frac{\epsilon\left(k_{r}-k_{m_{0}}\right)}{k_{r-1}} \\
& \leq \frac{1}{k_{r-1}}\left(\sup _{1 \leq i \leq m_{0}} H_{i} k_{m_{0}}\right)+\epsilon K<\frac{k_{m_{0}}}{k_{r-1}} T+\epsilon K
\end{aligned}
$$

from which we deduce that $x \in w[\mathcal{Z}]$.

Also; from theorem (2.3) and (2.4) follows; If $1<\liminf q_{r} \leq \lim \sup q_{r}<\infty$ then $N_{\theta}[\mathcal{Z}]=w[\mathcal{Z}]$.

\section{Lacunary statistical Zweier convergence}

The idea of statistical convergence was introduced by Fast [4] and studied by various authors (see [7], [8] and [3]).

A sequence $x=\left(x_{k}\right)$ is said to be statistically convergent to a number $s$ if for every $\epsilon>0$,

$$
\lim _{n \rightarrow \infty} \frac{1}{n}\left|\left\{k \leq n:\left|x_{k}-\ell\right| \geq \epsilon\right\}\right|=0,
$$

where the vertical bars indicate the number of elements in the enclosed set. In this case, we write $s t-\lim x=\ell$ and $S$ denotes the set of all statistical convergent sequences. 
A sequence $x=\left(x_{k}\right)$ is said to be lacunary statistical Zweier convergent to an $\ell$ if for $\epsilon>0$

$$
S_{\theta}[\mathcal{Z}]=\left\{x=\left(x_{i}\right) \in w: \lim _{r} \frac{1}{h_{r}}\left|Z K_{\theta}(\epsilon)\right|=0\right\},
$$

where $Z K_{\theta}(\epsilon)=\left\{i \in I_{r}:\left|\frac{1}{2}\left(x_{i}+x_{i-1}\right)-\ell\right| \geq \epsilon\right\}$.

If $x \in S_{\theta}[\mathcal{Z}]$, then we will write $x_{k} \rightarrow s\left(S_{\theta}[\mathcal{Z}]\right)$. Let

$$
I_{r}^{1}=\left\{i \in I_{r}:\left|\frac{1}{2}\left(x_{i}+x_{i-1}\right)-\ell\right| \geq \epsilon\right\}=C K_{\theta}(\epsilon)
$$

and

$$
I_{r}^{2}=\left\{i \in I_{r}:\left|\frac{1}{2}\left(x_{i}+x_{i-1}\right)-\ell\right|<\epsilon\right\} .
$$

Theorem 3.1. If $x_{i} \rightarrow s\left(N_{\theta}[\mathcal{Z}]\right)$, then $x_{i} \rightarrow s\left(S_{\theta}[\mathcal{Z}]\right)$.

Proof. If $\epsilon>0$ and $x_{i} \rightarrow s\left(N_{\theta}[\mathcal{Z}]\right)$, then we can write

$$
\frac{1}{h_{r}} \sum_{i \in I_{r}}\left|\frac{1}{2}\left(x_{i}+x_{i-1}\right)-\ell\right| \geq \frac{1}{h_{r}} \sum_{i \in I_{r}^{1}}\left|\frac{1}{2}\left(x_{i}+x_{i-1}\right)-\ell\right| \geq \frac{1}{h_{r}}\left|Z K_{\theta}(\epsilon)\right| \epsilon .
$$

It follows that $x_{i} \rightarrow s\left(S_{\theta}[\mathcal{Z}]\right)$.

Theorem 3.2. If $x \in N_{\infty}[\mathcal{Z}]$ and $x_{i} \rightarrow s\left(N_{\theta}[\mathcal{Z}]\right)$, then $x_{i} \rightarrow s\left(S_{\theta}[\mathcal{Z}]\right)$.

Proof. Suppose that $x \in N_{\infty}[\mathcal{Z}]$ and $x_{i} \rightarrow s\left(S_{\theta}[\mathcal{Z}]\right)$. Since $\sup \left|y_{i}\right|<\infty$, there is a constant $T>0$ such that $\left|\frac{1}{2}\left(x_{i}+x_{i-1}\right)-\ell\right|<T$ for all $i$. Therefore we have, for every $\epsilon>0$, that

$$
\begin{aligned}
& \frac{1}{h_{r}} \sum_{i \in I_{r}}\left|\frac{1}{2}\left(x_{i}+x_{i-1}\right)-\ell\right| \\
= & \frac{1}{h_{r}} \sum_{i \in I_{r}^{1}}\left|\frac{1}{2}\left(x_{i}+x_{i-1}\right)-\ell\right|+\frac{1}{h_{r}} \sum_{i \in I_{r}^{2}}\left|\frac{1}{2}\left(x_{i}+x_{i-1}\right)-\ell\right| \\
\leq & \frac{T}{h_{r}}\left|Z K_{\theta}(\epsilon)\right|+\epsilon .
\end{aligned}
$$

Taking limit as $\epsilon \rightarrow 0$, the desired result follows.

Theorem 3.3. If $x \in N_{\infty}[\mathcal{Z}]$, then we have $S_{\theta}[\mathcal{Z}]=N_{\theta}[\mathcal{Z}]$.

Proof. Proof follows from Theorem 3.1 and Theorem 3.2.

\section{References}

[1] J. Boos, Classical and Modern Methods in Summability, Oxford University Press, 2000.

[2] B. Cohuldhary and S. Nanda, Functional Analysis with Applications, Jhon Wiley \& Sons Inc, New Delhi.

[3] J. S. Connor, The statistical and strong p-Cesàro convergence spaces, Analysis 8 (1988), 47-63.

[4] H. Fast, Sur la convergence statistique, Colloq. Math. 2 (1950), 241-244. 
[5] A. R. Freedman, Some Cesàro-type summability spaces, Pasific J. Math. 35 (1981), 293-305.

[6] A. R. Freedman, J. J. Sember, and M. Raphael, Some Cesàro-type summability spaces, Proc. London Math. Soc. 37(1978), no. 3, 508-520.

[7] J. A. Fridy, On statistical convergence in Banach spaces, Analysis 5 (1985), 309-313.

[8] T. Salt, On statistically convergent sequences numbers, Math. Slovaca 30 (1980), 139150 .

[9] M. Şengönül, On The Zweier Space, Demonstratio Mathematica, vol. XL, No. 1, pp. 181-186, January 2005.

[10] A. Wilansky, Summability through Functional Analysis, North-Holland Matematics Studies 85 Amsterdam-Newyork-Oxfort, 1984.

Department of Mathematics

NEVŞEHIR UNIVERSITY

NevŞEHIR 50000, TürkiYE

E-mail address: msengonul@yahoo.com 